\title{
Covalent post-assembly modification triggers multiple structural transformations of a tetrazine-edged $\mathrm{Fe}_{4} \mathrm{~L}_{6}$ tetrahedron
}

\author{
Derrick A. Roberts ${ }^{\ddagger}$, Ben S. Pilgrim ${ }^{\ddagger}$, Giedre Sirvinskaite, Tanya K. Ronson, and Jonathan R. Nitschke* \\ Department of Chemistry, University of Cambridge, Lensfield Road CB2 1EW Cambridge, UK
}

\begin{abstract}
Covalent post-assembly modification (PAM) reactions are useful synthetic tools for functionalizing and stabilizing self-assembled metal-organic complexes. Recently, PAM reactions have also been explored as stimuli for triggering supramolecular structural transformations. Herein we demonstrate the use of inverse electron-demand Diels-Alder (IEDDA) PAM reactions to induce supramolecular structural transformations starting from a tetrazine-edged $\mathrm{Fe}^{\mathrm{II}}{ }_{4} \mathrm{~L}_{6}$ tetrahedral precursor. Following PAM, this tetrahedron rearranged to form three different architectures depending on the addition of other stimuli - an electron-rich aniline or a templating anion. By tracing the stimulus-response relationships within the system, we deciphered a network of transformations that mapped different combinations of stimuli onto specific transformation products. Given the many functions being developed for self-assembled three-dimensional architectures, this newly-established ability to control the interconversion between structures using combinations of different stimulus types may serve as the basis for switching the functions expressed within a system.
\end{abstract}

\section{INTRODUCTION}

The functions of many proteins are regulated through well-defined structural transformations in response to signals. ${ }^{1}$ These transformations range from local conformational changes to whole-protein reorganization. A diverse array of biological phenomena are thus enabled, including gas binding, catalysis, vision, and intracellular transport. ${ }^{2}$ Many stimuli can trigger these transformations, including light absorption, guest binding, and covalent post-translational modifications. Efforts to synthetically emulate biological structural transformations have led to insights into the mechanisms of these natural processes, while also informing the designs of artificial stimuli-responsive molecules and materials. ${ }^{3}$ Consequently, supramolecular structural transformations have been used to construct novel switchable catalysts, ${ }^{4}$ guest capture-and-release systems, ${ }^{5}$ responsive soft materials, ${ }^{6}$ and molecular mechanical actuators. ${ }^{7}$

Various stimuli have been used to control supramolecular structural transformations, including guest binding, ${ }^{8}$ ligand ${ }^{9}$ and metal ${ }^{10}$ substitution, photo-isomerization reactions, ${ }^{11}$ and environmental changes (e.g., $\mathrm{pH}$, temperature, and concentration). ${ }^{12}$ There are, however, few reports that use ligand-centered covalent post-assembly modification (PAM $)^{13}$ reactions to drive supramolecular transformations. ${ }^{14}$ One reason for the uncommon use of PAM in this context may be the narrow range of covalent reactions that cleanly functionalize metallosupramolecular structures without compromising their stabilities. Recently, however, new PAM strategies have provided mild and modular ways to covalently derivatize ${ }^{15}$ and stabilize ${ }^{16}$ self-assembled complexes, and to generate complex molecular topologies. ${ }^{17}$ These new PAM protocols can alter the electronic and steric parameters of self-assembled complexes. Such alterations may induce, in turn, well-defined structural transformations.
In this study, we develop inverse electron-demand Diels-Alder (IEDDA) PAM reactions to trigger the rearrangement of self-assembled metallocomplexes. Tetrazine-edged $\mathrm{Fe}^{\mathrm{II}} \mathrm{L}_{6}$ tetrahedron $\mathbf{1 a}$ (Figure 1) is the point of departure for all the transformations described herein. It was designed to react efficiently with alkyne dienophiles to afford the corresponding pyridazine-edged tetrahedron. ${ }^{18}$ Our investigations into the reactivity of $\mathbf{1 a}$ towards cyclooctyne in $\mathrm{CH}_{3} \mathrm{CN}$ revealed a sequence of structural rearrangements that led to the formation of three different cyclooctylpyridazine-edged architectures: $\mathrm{Fe}^{\mathrm{II}}{ }_{4} \mathrm{~L}_{6}$ tetrahedron 2 , $\mathrm{Fe}^{\mathrm{II}}{ }_{2} \mathrm{~L}_{3}$ helicate 3 , and $\mathrm{Fe}^{\mathrm{II}}{ }_{8} \mathrm{~L}_{12}$ twistedsquare-prism 4 (Figure 1). Additional stimuli, 4-methoxyaniline and $\mathrm{PF}_{6}{ }^{-}$, were observed to induce structures within the network to reassemble in different ways via orthogonal subcomponent exchange (SE) and anion templation (AT) pathways, respectively. Detailed analysis of the individual stimulus-response relationships enabled us to decipher how each combination of stimuli drives the system to a different major transformation product. Using this information, we constructed a hierarchical network that maps each architecture onto the stimuli that led to its formation (Figure 1). Thus, different architectures within the system could be induced to interconvert by combining covalent PAM with SE and AT co-stimuli in a sequence-dependent manner.

\section{RESULTS AND DISCUSSION}

Covalent PAM gates structural transformations. In the transformation network of Figure 1, covalent PAM is the primary stimulus that activates tetrazine-edged $\mathbf{1 a}$ towards subsequent structural rearrangements. Control experiments revealed that neither SE nor AT could drive transformation of $\mathbf{1 a}$ in the absence of PAM (see Supporting Information (SI), Section S5 for details). Thus, covalent PAM is the gateway stimulus for the network, producing a critical change in the ligand framework of tetrahedron $\mathbf{1}$ that renders it susceptible to structural rearrangements. 


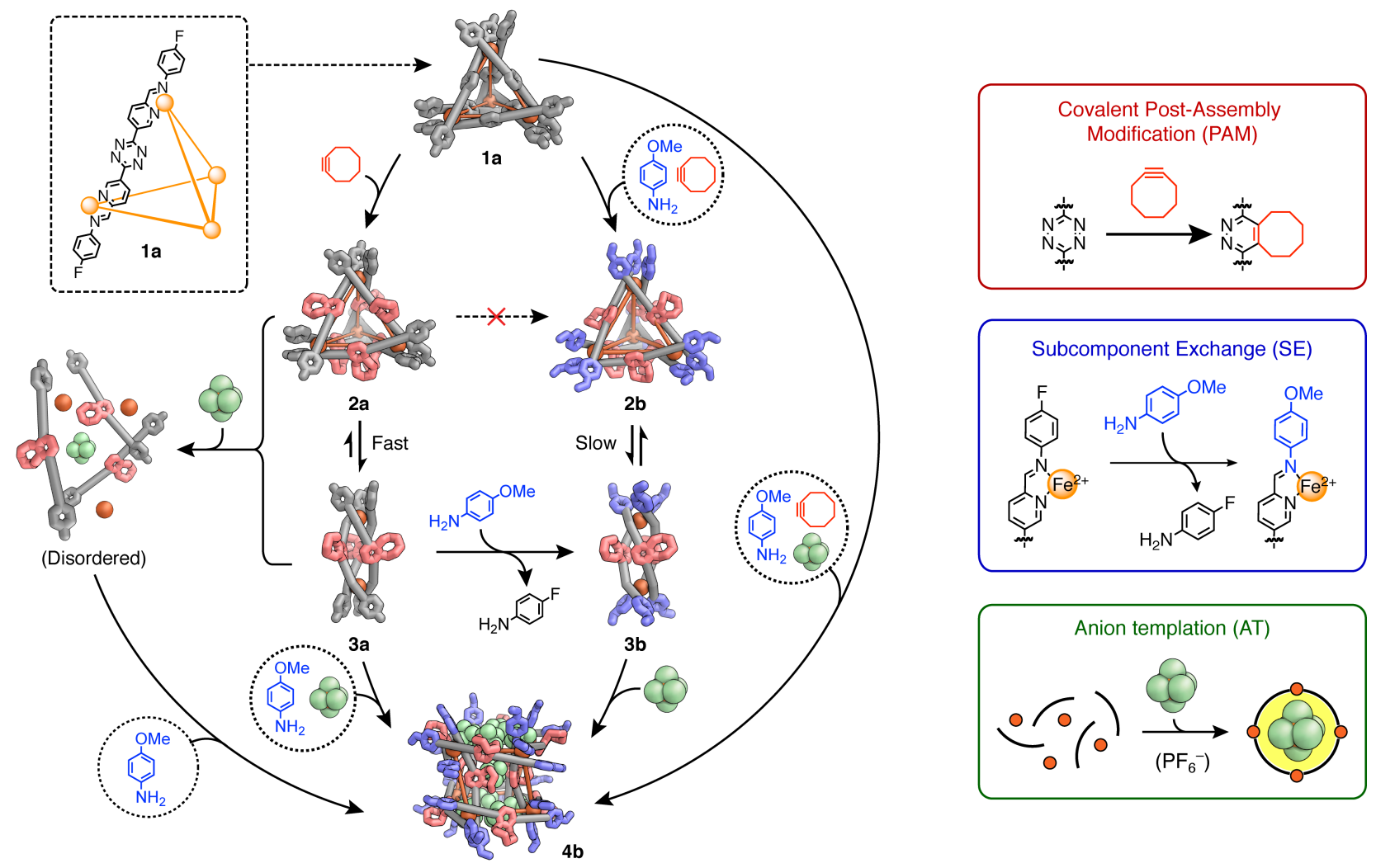

Figure 1. Stimulus-response map depicting the major products expressed by the system following the addition of different stimulus combinations.

Covalent PAM of 1 a was studied by in situ ${ }^{1} \mathrm{H}$ NMR spectroscopy. Treating 1a with cyclooctyne in $\mathrm{CD}_{3} \mathrm{CN}$ at $293 \mathrm{~K}$ resulted in the rapid $(<20 \mathrm{~min})$ formation of cyclooctylpyridazine-edged tetrahedron $\mathbf{2 a}$. The ${ }^{1} \mathrm{H}$ NMR spectrum of $\mathbf{2 a}$ confirmed its $T$-symmetric tetrahedral configuration, and DOSY NMR indicated a slightly larger hydrodynamic radius than 1a (Figure 2d). Further NMR analysis revealed that $\mathbf{2 a}$ was metastable, however, undergoing rearrangement into a new species over $1-2 \mathrm{~h}$ at room temperature. Heating $2 \mathbf{a}$ to $333 \mathrm{~K}$ for $2 \mathrm{~h}$ drove this rearrangement to completion, affording a single species, 3a, with a smaller DOSY hydrodynamic radius than either 1a or 2a. High-resolution ion mobility/ESI mass spectrometry (IM-MS) analysis revealed 3a to have an $\mathrm{Fe}^{\mathrm{II}}{ }_{2} \mathrm{~L}_{3}$ stoichiometry consistent with a dinuclear triple helicate structure (SI, Section S6). MM2 molecular mechanics modeling of 3a in both chiral (with both metal centers having the same configuration) and achiral/meso (with metal centers having opposing configurations) variants predicted strained structures requiring significant bending of the ligands bridging the iron(II) ions (SI, Figure S23).

The strained geometry of helicate $\mathbf{3 a}$ was reflected in its ${ }^{1} \mathrm{H}$ NMR spectrum: signals corresponding to $H^{1}$ and $H^{4}$ were broad and shifted downfield (Figure 2c) compared to analogous protons in tetrahedra 1a and 2a (Figure 2a, b). VT-NMR analysis of $\mathbf{3 a}$ in $\mathrm{CD}_{3} \mathrm{CN}$ revealed that a proportion of the iron(II) centers undergo a transition to a high spin state as the temperature of the solution increased from $243 \mathrm{~K}$ to $333 \mathrm{~K}$ (SI, Figure S24). This incipient spincrossover behavior is consistent with the strained geometry of the helicate. ${ }^{19}$ We hypothesize that the $\mathrm{N} \rightarrow \mathrm{Fe}$ bonds elongate progressively at higher temperatures to relieve ligand strain. This effect weakens the iron(II) ligand field, leading to a higher proportion of high-spin metal centers that contribute to the downfield shifts and broadening of the $H^{1}$ and $H^{4}$ signals.
After several hours at room temperature, helicate 3a partially reverted to tetrahedron $\mathbf{2 a}$, indicating a dynamic equilibrium between these complexes, $\mathbf{3} \mathbf{a}+\mathbf{3} \mathbf{a} \rightleftharpoons \mathbf{2} \mathbf{a}$, with an equilibrium constant $(K)$ :

$$
K=\frac{[2 a]}{[3 a]^{2}}
$$

Similar helicate-tetrahedron equilibria have been observed in other bis-bidentate ligand systems, ${ }^{20}$ including geometrically strained 2-iminopyridine chelates. ${ }^{21}$ Variable temperature ${ }^{1} \mathrm{H}$ NMR experiments allowed us to measure the temperature dependence of $K$ between $293 \mathrm{~K}$ and $333 \mathrm{~K}$ (Figure 3a); below $293 \mathrm{~K}$, the rate of equilibration was too slow to permit analysis (SI, Figure S25). Provided that the standard enthalpy and entropy changes of interconversion reaction are constant over this temperature range, Van 't Hoff analysis of these data (Figure $3 \mathrm{~b}$ ) gave:

$$
\begin{aligned}
& \Delta H^{\ominus}=-(54 \pm 2) \mathrm{kJ} \mathrm{mol}^{-1} \\
& \Delta S^{\ominus}=-(134 \pm 5) \mathrm{J} \mathrm{K}^{-1} \mathrm{~mol}^{-1}
\end{aligned}
$$

The relief of ligand strain when converting from helicate to tetrahedron is thus enthalpically favorable, while the loss of translational freedom due to fusion of two smaller complexes into a single larger one is entropically costly.

To assess which complex the system preferentially expresses at equilibrium, we defined $\mu_{\mathrm{sys}, \mathrm{F}}$ as the proportion of building blocks present in the helicate and tetrahedron populations:

$$
\mu_{s y s, \mathrm{~F}}=\frac{2[\mathbf{2} \boldsymbol{a}]}{[\mathbf{3 a}]}
$$

We have employed $\mu_{\text {sys,F }}$ rather than the canonical thermodynamic definition of favorability (i.e., the sign of $\Delta G^{\ominus}$ ) because, at the concentrations employed in these experiments $([\mathbf{1 a}] \approx 1.5 \mathrm{mM})$, over $99.5 \%$ of the system's building blocks must be converted into 
helicate 3a before $\Delta G^{\ominus}=0$ and there is a switchover in which side of the equilibrium is favored (SI, Section S6.5). As this conversion will happen only above $404 \mathrm{~K}$, which exceeds the boiling point of $\mathrm{CH}_{3} \mathrm{CN}$, it is more useful to consider the extent to which the system's building blocks are distributed between helicate and tetrahedron populations.

According to Equation 2, more than half of the system's building blocks constitute helicates when $\mu_{\text {sys,F }}<1$. This threshold was found at approximately $270 \mathrm{~K}$ at the concentrations employed herein (SI, Section S6.4). Above this temperature, the system is thus said to preferentially express helicate 3a over tetrahedron $\mathbf{2 a}$.

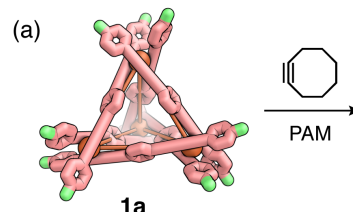

$1 a$

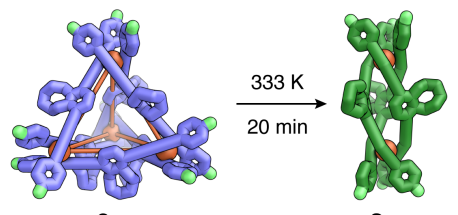

3a
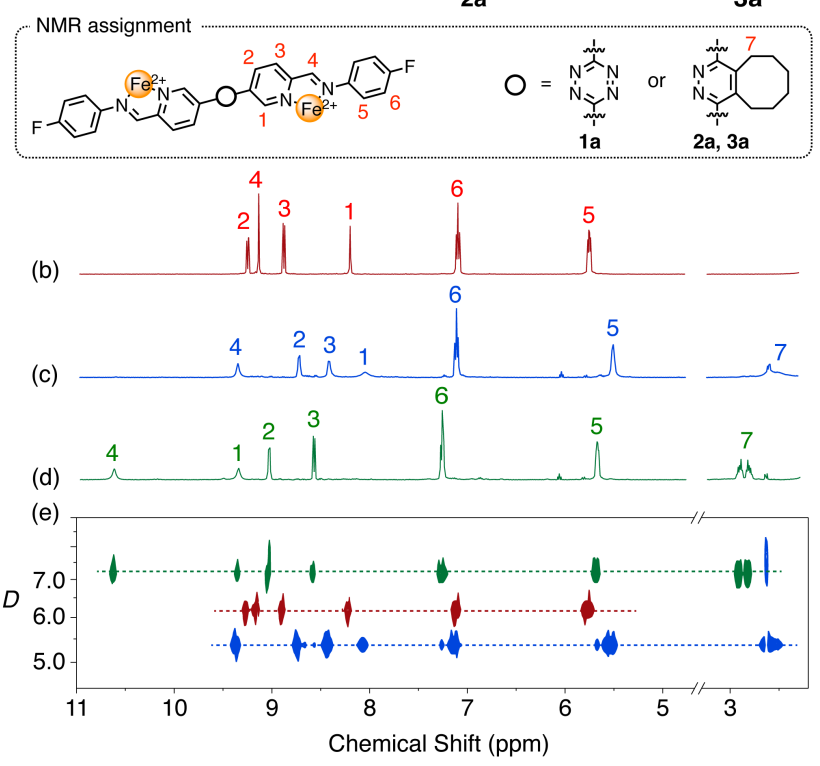

Figure 2. (a) The transformations of $\mathbf{1}$ a following treatment with cyclooctyne were followed by NMR: ${ }^{1} \mathrm{H}$ spectra of (b) Tetrahedron 1a (1.5 mM); (c) Tetrahedron 2a (40 min); (d) Helicate 3a after equilibration at $333 \mathrm{~K}$ for $2 \mathrm{~h}$ (conversion complete within $20 \mathrm{~min}$ ). (e) Overlaid ${ }^{1} \mathrm{H}$ DOSY NMR spectra of 1a, 2a and 3a, corresponding to spectra (b) -(d). The units of $D$ are $10^{-6} \mathrm{~cm}^{2} \mathrm{~s}^{-1}$.

Combining covalent PAM with subcomponent exchange. We hypothesized that substitution of the electron-poor 4-fluoroaniline residues within tetrahedron 1 a by more electron-rich 4-methoxyaniline would increase the strength of the metal-ligand bonds and thus influence the supramolecular product distribution following PAM-induced rearrangement. The reaction of tetrahedron 1a with 4-methoxyaniline furnished tetrahedron $\mathbf{1 b}$ (SI, Section S7). As with $\mathbf{1 a}, \mathbf{1} \mathbf{b}$ reacted with cyclooctyne (1.3 equiv. per tetrazine) in $\mathrm{CD}_{3} \mathrm{CN}$ at $293 \mathrm{~K}$ to form cycloocytylpyridazine-edged tetrahedron 2b (Figure 4 b). Tetrahedron $\mathbf{2 b}$ also existed in equilibrium with $\mathrm{Fe}^{\mathrm{II}}{ }_{2} \mathrm{~L}_{3}$ helicate $\mathbf{3 b}$. However, the rate of interconversion between $\mathbf{2 b}$ and $\mathbf{3 b}$ was appreciably slower due to the stronger $\mathrm{N} \rightarrow$ Fe bonds, allowing $\mathbf{2} \mathbf{b}$ to persist as the dominant product for several hours at $293 \mathrm{~K}$ following PAM. This property permitted the analysis of both complexes by IM-MS, which revealed significantly different drift times in the gas phase (SI, Section S7). Heating the reaction mixture to $333 \mathrm{~K}$ for $2 \mathrm{~h}$ resulted in near-complete conversion to helicate $3 \mathrm{~b}$ (Figure 4c).
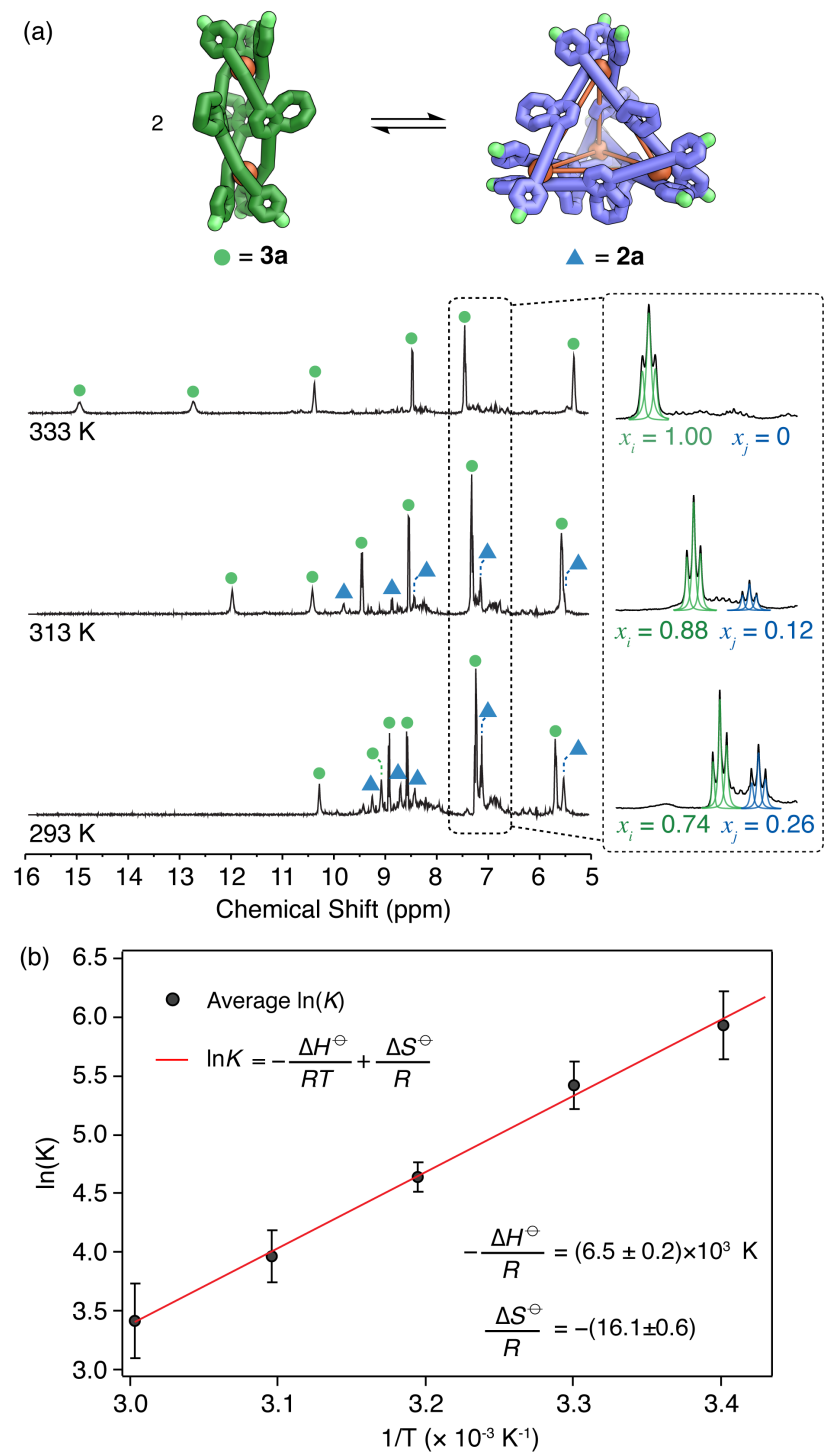

Figure 3. Analysis of the temperature dependence of the equilibrium between tetrahedron $2 \mathbf{a}$ and helicate 3a. (a) ${ }^{1} \mathrm{H}$ NMR spectra of a mixture of $\mathbf{2} \mathbf{a}$ and $\mathbf{3} \mathbf{a}$ at different temperatures, with incipient spin-crossover behavior evident in 3a and, to a lesser extent, in 2a. (b) Van 't Hoff analysis of the equilibrium.

The stronger $\mathrm{N} \rightarrow \mathrm{Fe}$ bonds in the structures containing methoxyaniline residues also influenced the position of the helicate-tetrahedron equilibrium. Even after equilibration at $333 \mathrm{~K}$ for $2 \mathrm{~h}$, peaks corresponding to tetrahedron $\mathbf{2 b}$ were still visible by NMR (Figure 4c). By contrast, the fluoroaniline system produced exclusively helicate under similar conditions (Figure 3a). Incorporation of an electron-rich aniline thus shifted the helicate-tetrahedron equilibrium in favor of the tetrahedron. This shift was also apparent at lower temperatures, although the slower rate of interconversion precluded quantitative study. We infer helicate formation to be less favorable in the methoxyaniline system because the shorter $\mathrm{N} \rightarrow \mathrm{Fe}$ bonds would exacerbate the ligand strain involved in helicate formation. 
Unexpectedly, SE of the 2a/3a mixture with 4-methoxyaniline at $278 \mathrm{~K}$ initially led to disproportionately greater formation of helicate $\mathbf{3} \mathbf{b}$ than of tetrahedron $\mathbf{2} \mathbf{b}$. A post-PAM initial $\mu_{\mathrm{sys}, \mathrm{F}}$ of 2.3 was obtained, as determined by ${ }^{1} \mathrm{H}$ NMR (SI, Figure S33), indicating that more than twice the number of subcomponents were bound up in the tetrahedron 2a population than in the helicate 3a population. The SE reaction of 4-methoxyaniline with this mixture at $278 \mathrm{~K}$ proceeded slowly, requiring 7 days to approach completion. Helicate $\mathbf{3 b}$ was initially observed to predominate over tetrahedron $\mathbf{2 b}$, with an initial $\mu_{\text {sys, OMe }}$ of the system of only 0.25 , marking $\mathbf{3 b}$ as the kinetic product of the transformation.

$$
\mu_{s y s, \mathrm{OMe}}=\frac{2[\mathbf{2} \boldsymbol{b}]}{[\mathbf{3} \boldsymbol{b}]}
$$

The $\mathbf{2 b} / \mathbf{3 b}$ mixture then underwent slow rearrangement to express more tetrahedron $\mathbf{2 b}$. This process required 3 weeks to approach equilibrium at $278 \mathrm{~K}$, however.

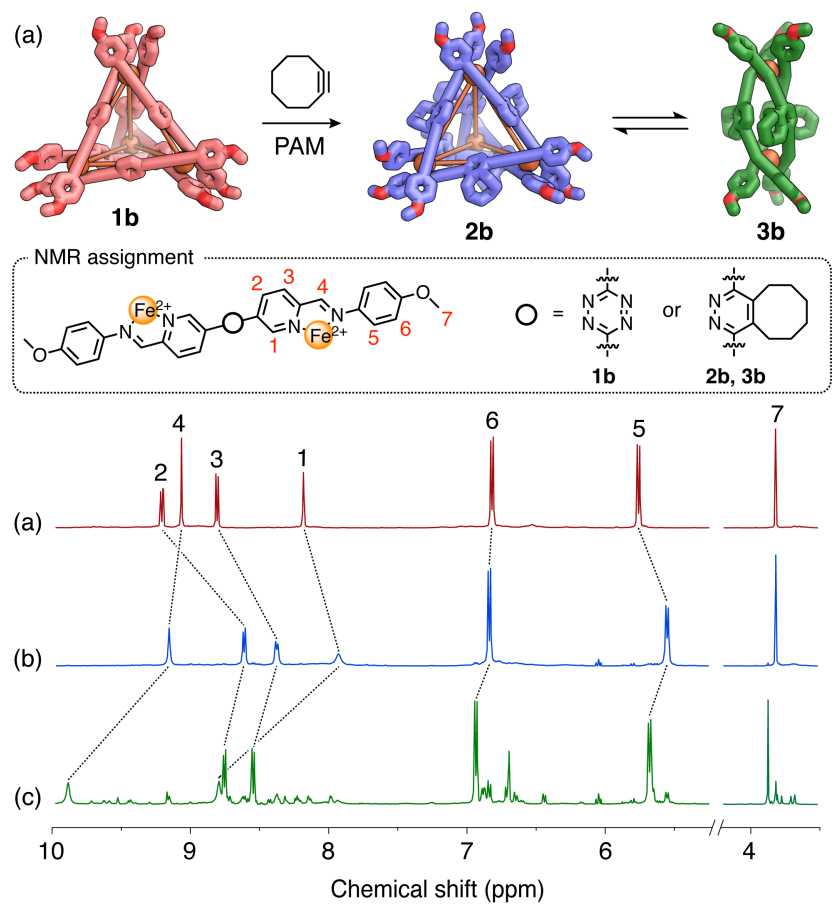

Figure 4. (a) Transformations of $\mathbf{1 b}$ following treatment with cyclooctyne were followed by NMR: ${ }^{1} \mathrm{H}$ spectra of (b) Tetrahedron $\mathbf{1 b}$ $(1.5 \mathrm{mM})$; (c) Tetrahedron $\mathbf{2 b}$, recorded $2 \mathrm{~h}$ after addition of cyclooctyne; (d) Helicate $3 \mathbf{b}$, recorded after equilibration at $333 \mathrm{~K}$ for $2 \mathrm{~h}$.

These results suggest that SE proceeds more rapidly for helicate 3a than for tetrahedron 2a. We infer the principal route of SE thus to consist of conversion of helicate $\mathbf{3 a}$ into helicate $\mathbf{3 b}$. Equilibration between $\mathbf{2} \mathbf{a}$ and 3a then regenerates more 3a, leading to further amine substitution via the helicate rather than the tetrahedron. Once formed, the stronger $\mathrm{N} \rightarrow$ Fe bonds in helicate $\mathbf{3} \mathbf{b}$ stall its conversion into $\mathbf{2 b}$ at lower temperatures, allowing it to build up as an intermediate at $278 \mathrm{~K}$.

The higher rate of helicate amine substitution can be understood in terms of the longer and weaker $\mathrm{N} \rightarrow \mathrm{Fe}$ bonds in the strained helicate structure, enabling faster SE than in the less-strained tetrahedron. These observations agree with recent findings by Hooley and co-workers, which demonstrated how ligand strain can drastically influence amine substitution rates - and, thus, supramolecular structural outcomes-in imine-containing metallocomplexes. ${ }^{22}$
Deciphering the effects of three chemical stimuli. The transformation network has so far been shown to respond to PAM and SE stimuli to express tetrahedral and helicate structures. Targeting more complex, higher-nuclearity architectures, however, requires the $\mathrm{Fe}^{\mathrm{II}}$ centers to adopt lower-symmetry meridional (mer) configurations as opposed to higher-symmetry facial ( $f a c)$ stereochemistry. The presence of templating anions was observed to drive such a facto-mer transition in this system, leading to the formation of a larger complex, twisted square prism 4 , containing exclusively mer-configured $\mathrm{Fe}^{\mathrm{II}}$ vertices.

We first observed prism 4 when attempting to crystallize helicate 3a. Single crystals suitable for $\mathrm{X}$-ray diffraction analysis were obtained by the slow diffusion of benzene vapor into an acetonitrile solution of $\mathbf{2 a}$ and $\mathbf{3 a}$ containing $\mathrm{Bu}_{4} \mathrm{NPF}_{6}$ ( 50 equiv). We were initially surprised when $\mathrm{X}$-ray analysis revealed $\mathrm{Fe}^{\mathrm{II}}{ }_{8} \mathrm{~L}_{12}$ twisted square prism complex $4 \mathbf{a}$ (Figure 5) rather than the anticipated $\mathrm{Fe}^{\mathrm{II}}{ }_{2} \mathrm{~L}_{3}$ helicate.

Prism 4 a consists of two four-sided circular helicate rings containing four metal centers and four equatorial ligands. The two square rings are bridged by four axial ligands, and are twisted by $29 \pm 2^{\circ}$ with respect to each other (Figure $5 \mathrm{~b}$ ). The average metalto-metal distances both within and between the $\mathrm{Fe}^{\mathrm{II}}{ }_{4} \mathrm{~L}_{4}$ rings are very similar $(11.7 \pm 0.3 \AA)$. All metal centers possess the same $\Delta$ or $\Lambda$ stereochemistry within a given complex, lending the structure idealized $D_{4}$ point symmetry. Both enantiomers of $4 \mathbf{a}$ were present in the crystal. The angles between the pyridine-pyridazine-pyridine ring centroids were $161-173^{\circ}$ and the dihedral angles between the pyridine and pyridazine rings were $41-63^{\circ}$, indicating the presence of bending and twisting away from a linear and coplanar orientation.
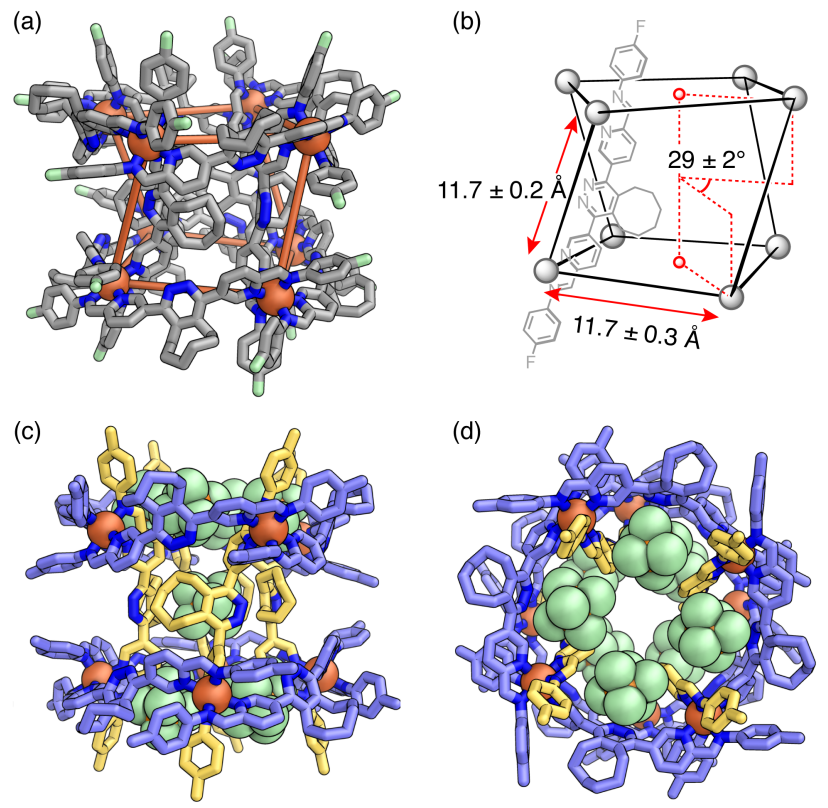

Figure 5. Representations of the X-ray crystal structure of $\mathrm{PF}_{6}{ }^{-} \subset \mathbf{4 a}$. Hydrogen atoms, disorder, solvent molecules and non-encapsulated counterions are omitted for clarity. (a) 4 a shown with its $\mathrm{Fe}^{\mathrm{II}}$ framework highlighted in orange. (Key: gray $=$ carbon, blue $=$ nitrogen, orange $=$ iron, green $=$ fluorine $)$. (b) Schematic structure of $4 \mathbf{a}$ illustrating average $\mathrm{Fe}-\mathrm{Fe}$ distances and the twist angle between the square faces of the prism. (c) Side view of $\mathbf{4 a}$ highlighting the positions of bound $\mathrm{PF}_{6}{ }^{-}$ions. Equatorial and axial ligands are colored blue and yellow, respectively. (d) View along the $C_{4}$ axis of $\mathbf{4 a}$, highlighting counter-ions bound around the upper and lower rims of the prism. The central $\mathrm{PF}_{6}{ }^{-}$ion is omitted in (d) to highlight the channel through the complex. 
Prism 4a encapsulated nine $\mathrm{PF}_{6}{ }^{-}$anions in the crystal. Eight of these are bound within partially-enclosed pockets within the two $\mathrm{Fe}^{\mathrm{II}}{ }_{4} \mathrm{~L}_{4}$ circular helicate rings, and the ninth is held within the central void of the prism. Close contacts between the cyclooctylpyridazine rings and the $\mathrm{PF}_{6}{ }^{-}$ions suggested that anion- $\pi$ interactions contribute to the stability of the observed host-guest complex. ${ }^{23}$

The encapsulation of nine $\mathrm{PF}_{6}{ }^{-}$anions within $\mathbf{4 a}$ in the solid state led us to infer that the addition of suitable templating anions could drive a helicate-to-prism transformation in solution. ${ }^{24}$ Treating a 2a/3a mixture with $\mathrm{PF}_{6}{ }^{-}, \mathrm{BF}_{4}{ }^{-}$or $\mathrm{ClO}_{4}{ }^{-}$led to the appearance of multiple new ${ }^{1} \mathrm{H}$ NMR signals, suggesting the formation of higher-nuclearity structures (SI, Figure S34). These structures might be larger prisms of the general formula $\mathrm{Fe}_{2 \mathrm{x}} \mathrm{L}_{3 \mathrm{x}}$ (where $\mathrm{x} \geq 4$ ). ${ }^{9 \mathrm{~b}, 25}$ However, these transformations were incomplete, yielding intractably complicated mixtures rather than a discrete major product.

In the case of $\mathrm{BF}_{4}{ }^{-}$, we obtained single crystals suitable for crystallography by slow diffusion of benzene vapor into a $\mathrm{CH}_{3} \mathrm{CN}$ solution of the initial transformation library. $\mathrm{X}$-ray diffraction analysis revealed the formation of $\mathrm{Fe}^{\mathrm{II}} \mathrm{L}_{12}$ prism $\mathrm{BF}_{4}{ }^{-} \subset \mathbf{4 a}$ as the only observed product, analogous to the $\mathrm{PF}_{6}{ }^{-}$inclusion complex. The binding pockets of $\mathrm{BF}_{4}{ }^{-} \subset \mathbf{4 a}$, however, exhibited partial occupancy with a mixture of $\mathrm{CH}_{3} \mathrm{CN}$ and $\mathrm{BF}_{4}{ }^{-}$guests (SI, Figure S64), suggesting that $\mathrm{BF}_{4}^{-}$is a poor fit for the binding pockets of 4 a compared to $\mathrm{PF}_{6}^{-}$.

The observation that $\mathbf{2 a}$ and $\mathbf{3 a}$ did not rearrange cleanly into twisted square prism $\mathbf{4 a}$ in solution suggests that AT alone could not drive this transformation. We infer that in the solid state, crystal packing provided the stabilization needed to selectively form $\mathbf{4 a}$ as the only observed product. We anticipated that combining AT with SE using more electron-rich 4-methoxyaniline could provide analogous stabilization in solution by increasing the strengths of the $\mathrm{N} \rightarrow \mathrm{Fe}$ bonds, thereby providing an additional enthalpic driving force for the formation of the larger $\mathrm{Fe}^{\mathrm{II}}{ }_{8} \mathrm{~L}_{12}$ architecture.

Heating a mixture of $\mathbf{2} \mathbf{b}$ and $\mathbf{3 b}$ with an excess of $\mathrm{Bu}_{4} \mathrm{NPF}_{6}$ (20 equiv) to $333 \mathrm{~K}$ for $16 \mathrm{~h}$ gave a single major product, as determined by ${ }^{1} \mathrm{H}$ NMR spectroscopy (Figure 6c). The spectrum of this species reflected the symmetry of prism $\mathbf{4 b}$, exhibiting three imine and three methoxy proton environments characteristic of mer-configured metal vertices (SI, Figure S36). ${ }^{1} \mathrm{H}$ DOSY NMR analysis also confirmed that this complex diffused at a slower rate than either tetrahedron $\mathbf{2 b}$ or helicate $\mathbf{3 b}$ (Figure $6 \mathrm{~d}$ ). Moreover, we observed excellent agreement between the calculated effective spherical radii of the three complexes and their DOSY-determined hydrodynamic radii (SI, Figure S35). Diffraction-quality single crystals of $\mathbf{4 b}$ were obtained by slow vapor diffusion of benzene into an acetonitrile solution of the complex containing excess $\mathrm{Bu}_{4} \mathrm{NPF}_{6}$ (50 equiv). X-ray crystallography established the structure of the square prism in the solid state, which was analogous to $\mathrm{PF}_{6}{ }^{-} \subset \mathbf{4 a}$ (SI, Figure S68).

Prism $\mathbf{4 b}$ was also prepared by combining pre-functionalised cyclooctylpyridazine dialdehyde (SI, Section S9), 4-methoxyaniline and $\mathrm{Fe}\left(\mathrm{PF}_{6}\right)_{2}$ in $\mathrm{CD}_{3} \mathrm{CN}$ at $333 \mathrm{~K}$ for $48 \mathrm{~h}$. Subsequent purification of the reaction mixture by size-exclusion chromatography (SEC) removed impurities to give a clean ${ }^{1} \mathrm{H}$ NMR spectrum of $\mathbf{4 b}$ for highresolution NMR analysis (Figure 7a). A small amount of helicate $\mathbf{3 b}$ co-eluted with $\mathbf{4 b}$, but was readily distinguishable (Figure $6 \mathrm{c}$ ). The ${ }^{1} \mathrm{H}$ NMR spectrum of purified $\mathbf{4} \mathbf{b}$ matched that of the product formed following the treatment of a mixture of $\mathbf{2} \mathbf{b}$ and $\mathbf{3} \mathbf{b}$ with $\mathrm{PF}_{6}{ }^{-}$.

Prism $\mathbf{4 b}$ was only obtained as the major product in solution when all three stimuli were present. We infer that AT, which provided stabilizing anion- $\pi$ interactions within the $\mathrm{Fe}^{\mathrm{II}}{ }_{8} \mathrm{~L}_{12}$ prism framework, ${ }^{26}$ and SE, which increased the strength of the $\mathrm{N} \rightarrow \mathrm{Fe}$ bonds, were both required to overcome the entropic cost of forming the larger $\mathrm{Fe}^{\mathrm{II}}{ }_{8} \mathrm{~L}_{12}$ architecture. Prior studies suggest that more electron-rich aniline residues produce stronger quadrupolar $\pi$-interactions between the aniline and pyridine rings at the mer-verticies, thus affording additional stabilization to the prism architecture. ${ }^{27}$

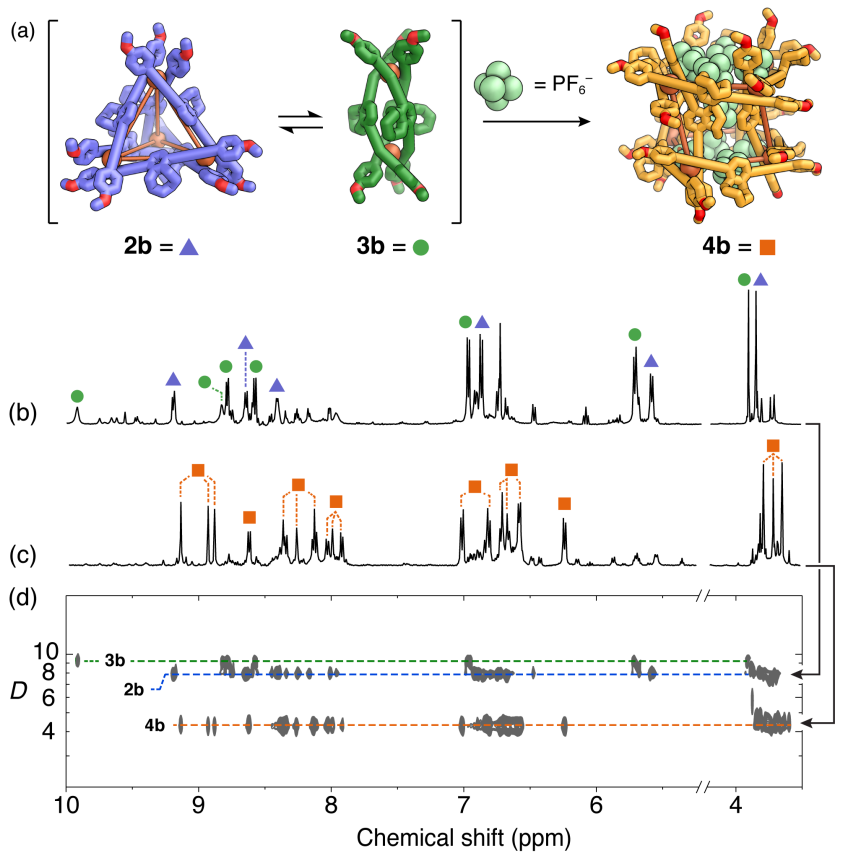

Figure 6. (a) Equilibration of a mixture of $\mathbf{2 b}$ and $\mathbf{3 b}$ at $293 \mathrm{~K}$ for $48 \mathrm{~h}$ gave rise to ${ }^{1} \mathrm{H}$ NMR spectrum (b) $\left(500 \mathrm{MHz}, 298 \mathrm{~K}, \mathrm{CD}_{3} \mathrm{CN}\right)$, which transformed into spectrum (c), corresponding to twisted square prism 4b, upon addition of $\mathrm{PF}_{6}{ }^{-}$. (d) Overlaid DOSY NMR spectra corresponding to spectra (b) and (c), as indicated by the arrows, comparing the diffusivities of $\mathbf{2} \mathbf{b}, \mathbf{3 b}$ and $\mathbf{4 b}$. The units of $D$ are $10^{-6} \mathrm{~cm}^{2} \mathrm{~s}^{-1}$.

Establishing a stimulus hierarchy via sequential transformations. In the above discussion, we explored individual steps within the transformation network to simplify our analysis of the overall system. It is, however, possible to traverse different paths within this network by the sequential addition of PAM, SE and AT stimuli (SI, Section S10). Importantly, carrying out transformations in sequence allowed us to ascertain the pathway dependence of the network and thus sort the three stimulus types in order of their ability to drive discrete transformations within the system.

Control experiments had demonstrated that no structural transformations were possible without first adding cyclooctyne, emphasizing the role of PAM as the primary stimulus. We hypothesize that the bulky cyclooctyl groups introduced through PAM caused the central ring of the ligands to adopt a non-planar conformation that promotes formation of the helicate and prism architectures. Ligand non-planarity, both in the dihedral angles between rings and in the angles between ring centroids, is noticeable in the crystal structures of $\mathbf{4 a}$ and $\mathbf{4 b}$ (SI, Section S11) and the optimized geometries of MM2 molecular models of helicates $\mathbf{3 a}$ and $\mathbf{3 b}$. Conversely, the tetrazine ligand does not have sufficient steric bulk to favor the ligand geometries that lead to the helicate and prism.

SE is the next most important stimulus since, when combined with PAM, it influenced the relative proportion of tetrahedron and 
helicate, as reflected in the different helicate $\rightleftharpoons$ tetrahedron equilibrium constants for the fluoroaniline and methoxyaniline-derived systems. Without initial PAM, however, SE was not observed to drive structural transformation. SE could, however, result in the conversion of tetrahedron $\mathbf{1 a}$ to $\mathbf{1 b}$. Finally, AT required the presence of both PAM and SE stimuli to influence the product distribution of the system in solution. Thus, the hierarchical ordering of the ability of the three stimuli to bring about change in the structure type is $\mathrm{PAM}>\mathrm{SE}>\mathrm{AT}$.

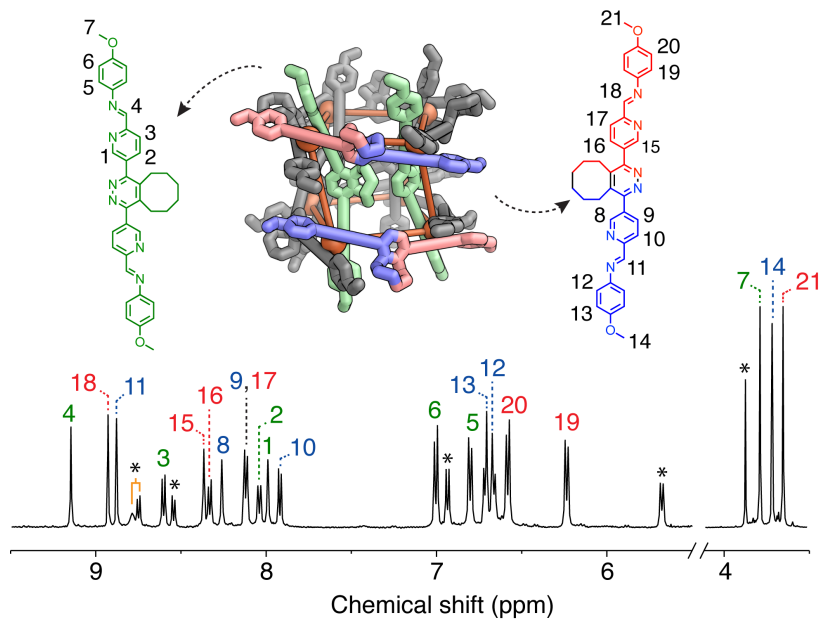

Figure 7. Assigned ${ }^{1} \mathrm{H}$ NMR spectrum $\left(500 \mathrm{MHz}, 298 \mathrm{~K}, \mathrm{CD}_{3} \mathrm{CN}\right)$ of prism $\mathbf{4 b}$, synthesized directly from building blocks and purified by size exclusion chromatography. A small amount of helicate $\mathbf{3 b}$ (marked with asterisks) co-eluted with prism $\mathbf{4 b}$.

While the stimulus combinations discussed above generated discrete products, one combination failed to produce a well-defined response at all. The addition of $\mathrm{PF}_{6}{ }^{-}$to a mixture of $\mathbf{2 a}$ and $\mathbf{3 a}$ led to the system moving towards a more disordered state, ${ }^{28}$ as evidenced by the broadness of its NMR spectrum (SI, Figure S59c). We attribute this disorder to the incipient formation of anion-templated structures, which nonetheless lack a sufficient driving force to produce a single discrete complex. However, completing the required set of stimuli by performing SE caused prism $\mathbf{4 b}$ to emerge from the disordered mixture as the sole product (SI, Figure S59d). This observation highlights the pathway-dependent nature of the network, whereby the order of stimulus addition does not affect the ultimate outcome $(\mathbf{4 b})$, but does determine what intermediate ordered states $(\mathbf{2 a}, \mathbf{3} \mathbf{a}, \mathbf{1 b}, \mathbf{2} \mathbf{b}$, or $\mathbf{3} \mathbf{b})$ and disordered states ${ }^{29}$ are expressed by the system along the way.

In addition to elucidating the structures expressed by the system after the various stimuli have been added, our investigations of these transformation sequences also provided insight into the relative rates of the various processes. PAM triggered a rapid structural transformation, with IEDDA proceeding to completion on both $\mathbf{1 a}$ and $\mathbf{1 b}$ within $20 \mathrm{~min}$ at $293 \mathrm{~K}$. Both the $\mathbf{2} \rightleftharpoons \mathbf{3}$ equilibria and subcomponent exchange are slower processes at $293 \mathrm{~K}$, taking hours to days, as discussed above. Anion templation was the slowest of these processes, as heating to $333 \mathrm{~K}$ was required for $24-48 \mathrm{~h}$ before prism $\mathbf{4 b}$ emerged as the dominant product.

\section{CONCLUSION}

Covalent PAM strategies are most often designed to preserve the structural integrity of supramolecular complexes. ${ }^{13}$ Inducing structural transformations is thus an emerging application of PAM that seeks to controllably introduce instability into self-assembled architecture through covalent reactions. It is a challenge to develop modifications that render a parent architecture sufficiently unstable to undergo a rearrangement, but not so unstable as to destroy it completely. In this contribution, we have demonstrated an approach using the tetrazine-based IEDDA reaction to induce transformations of a tetrazine-edged tetrahedral cage. The PAM stimulus (cyclooctyne) could be combined with additional co-stimuli (an electron rich aniline or an anion template) to influence the supramolecular product distribution, thereby rendering the system responsive to three unique stimulus types that operate together.

Different potential functions could be expressed by this system or its derivatives following the applications of various combinations of stimuli. Tetrahedral complexes can be employed as molecular containers that bind cargoes, ${ }^{30}$ whereas most helicates have minimal cavity volume for guest encapsulation but can interact with molecules such as DNA. ${ }^{31}$ The inner channels of prisms such as $\mathbf{4 b}$ enable them to act as pores through membranes. ${ }^{32}$ The ability to map different combinations of stimuli to unique structural outcomes, to switch from order in the system to disorder and back again, ${ }^{33}$ thus reveals opportunities for tailoring their functions to unique combinations of chemical stimuli.

\section{ASSOCIATED CONTENT}

\section{Supporting Information}

The Supporting Information is available free of charge on the ACS Publications website.

PDF Supporting information containing experimental procedures, spectroscopic data, MM2 modelling and X-ray crystallography (PDF).

$\mathrm{X}$-ray data for $\mathrm{PF}_{6}{ }^{-} \subset \mathbf{4 a}(\mathrm{CCDC} 1577717)$ (CIF).

$\mathrm{X}$-ray data for $\mathrm{BF}_{4}{ }^{-} \subset \mathbf{4 a}(\mathrm{CCDC} 1577718)$ (CIF).

$\mathrm{X}$-ray data for $\mathrm{PF}_{6}{ }^{-} \subset \mathbf{4 b}(\mathrm{CCDC} 1577719)$ (CIF).

\section{AUTHOR INFORMATION}

\section{Corresponding Author}

*jrn34@cam.ac.uk

\section{Author Contributions}

tThese authors contributed equally.

All authors have given approval to the final version of the manuscript.

\section{ACKNOWLEDGMENT}

D.A.R. acknowledges the Gates Cambridge Trust. B.S.P. acknowledges the Royal Commission for the Exhibition of 1851 Fellowship and Corpus Christi College, Cambridge. This work was supported by the UK Engineering and Physical Sciences Research Council (EP/M01083X/1). The authors thank Diamond Light Source (UK) (MT11397), University of Cambridge NMR facility, and the EPSRC UK National Mass Spectrometry Facility at Swansea University. The authors thank Dr Felix Rizzuto and Ms Marion Kieffer for recording HRMS and IM-MS data.

\section{REFERENCES}

1. Grant, B. J.; Gorfe, A. A.; McCammon, J. A., Curr. Opin. Struct. Biol. 2010, 20, 142-147.

2. Alberts, B.; Johnson, A.; Lewis, J.; Raff, M.; Roberts, K.; Walter, P., Molecular Biology of the Cell. 4th ed.; Garland Science: New York, 2002. 3. (a) Das, S.; Ranjan, P.; Maiti Pradipta, S.; Singh, G.; Leitus, G.; Klajn, R., Adv. Mater. 2012, 25, 422-426; (b) Dadon, Z.; Samiappan, M.; 
Wagner, N.; Ashkenasy, G., Chem. Commun. 2012, 48, 1419-1421; (c) Zhuang, J.; Gordon, M. R.; Ventura, J.; Li, L.; Thayumanavan, S., Chem. Soc. Rev. 2013, 42, 7421-7435; (d) Jie, K.; Zhou, Y.; Yao, Y.; Shi, B.; Huang, F., J. Am. Chem. Soc. 2015, 137, 10472-10475; (e) Lee, J.-W.; Klajn, R., Chem. Commun. 2015, 51, 2036-2039; (f) Nguyen, V. D.; Pal, A.; Snijkers, F.; Colomb-Delsuc, M.; Leonetti, G.; Otto, S.; van der Gucht, J., Soft Matter 2016, 12, 432-440; (g) Wang, W.; Wang, Y.-X.; Yang, H.-B., Chem. Soc. Rev. 2016, 45, 2656-2693; (h) Holub, J.; Vantomme, G.; Lehn, J.-M., J. Am. Chem. Soc. 2016, 138, 11783-11791; (i) Matysiak, B. M.; Nowak, P.; Cvrtila, I.; Pappas, C. G.; Liu, B.; Komáromy, D.; Otto, S., J. Am. Chem. Soc. 2017, 139, 6744-6751; (j) Shieh, Y.-T.; Hu, F.-Z.; Cheng, C.-C., ACS Applied Nano Materials 2018, 1, 384-393; (k) Daniels, G. C.; Camerino, E.; Wynne, J. H.; Iezzi, E. B., Materials Horizons 2018.

4. (a) Wiester, M. J.; Ulmann, P. A.; Mirkin, C. A., Angew. Chem., Int. Ed. 201 1, 50, 114-137; (b) Blanco, V.; Leigh, D. A.; Marcos, V., Chem. Soc. Rev. 2015, 44, 5341-5370.

5. (a) Han, M.; Michel, R.; He, B.; Chen, Y.-S.; Stalke, D.; John, M.; Clever, G. H., Angew. Chem., Int. Ed. 2013, 52, 1319-1323; (b) Kishi, N.; Akita, M.; Kamiya, M.; Hayashi, S.; Hsu, H.-F.; Yoshizawa, M., J. Am. Chem. Soc. 2013, 135, 12976-12979; (c) Preston, D.; Fox-Charles, A.; Lo, W. K. C.; Crowley, J. D., Chem. Commun. 2015, 51, 9042-9045.

6. (a) Krieg, E.; Shirman, E.; Weissman, H.; Shimoni, E.; Wolf, S. G.; Pinkas, I.; Rybtchinski, B., J. Am. Chem. Soc. 2009, 131, 14365-14373; (b) Wei, P.; Yan, X.; Huang, F., Chem. Soc. Rev. 2015, 44, 815-832; (c) Wei, S. C.; Pan, M.; Fan, Y. Z.; Liu, H.; Zhang, J.; Su, C. Y., Chem. Eur. J. 2015, 21, 7418-7427; (d) Zhang, W.; Gao, C., J. Mater. Chem. A 2017, 5, 16059-16104.

7. (a) Badjić, J. D.; Balzani, V.; Credi, A.; Silvi, S.; Stoddart, J. F., Science 2004, 303, 1845; (b) Kai, H.; Nara, S.; Kinbara, K.; Aida, T., J. Am. Chem. Soc. 2008, 130, 6725-6727; (c) Durola, F.; Heitz, V.; Reviriego, F.; Roche, C.; Sauvage, J.-P.; Sour, A.; Trolez, Y., Acc. Chem. Res. 2014, 47, 633-645.

8. (a) Umemoto, K.; Yamaguchi, K.; Fujita, M., J. Am. Chem. Soc. 2000, 122, 7150-7151; (b) Yamanoi, Y.; Sakamoto, Y.; Kusukawa, T.; Fujita, M.; Sakamoto, S.; Yamaguchi, K., J. Am. Chem. Soc. 2001, 123, 980-981; (c) Kumazawa, K.; Yamanoi, Y.; Yoshizawa, M.; Kusukawa, T.; Fujita, M., Angew. Chem., Int. Ed. 2004, 43, 5936-5940; (d) Wood, D. M.; Meng, W.; Ronson, T. K.; Stefankiewicz, A. R.; Sanders, J. K. M.; Nitschke, J. R., Angew. Chem., Int. Ed. 2015, 54, 3988-3992; (e) Lee, H.; Elumalai, P.; Singh, N.; Kim, H.; Lee, S. U.; Chi, K.-W., J. Am. Chem. Soc. 2015, 137, 4674-4677; (f) Wang, B.; Zang, Z.; Wang, H.; Dou, W.; Tang, X.; Liu, W.; Shao, Y.; Ma, J.; Li, Y.; Zhou, J., Angew. Chem., Int. Ed. 2013, 52, 3756-3759.

9. (a) Neogi, S.; Lorenz, Y.; Engeser, M.; Samanta, D.; Schmittel, M., Inorg. Chem. 2013, 52, 6975-6984; (b) Meng, W.; Ronson, T. K.; Clegg, J. K.; Nitschke, J. R., Angew. Chem., Int. Ed. 2013, 52, 1017-1021; (c) Zhou, X.-P.; Wu, Y.; Li, D., J. Am. Chem. Soc. 2013, 135, 16062-16065; (d) Samanta, D.; Mukherjee, P. S., Chem. Eur. J. 2014, 20, 12483-12492. 10. (a) Carnes, M. E.; Collins, M. S.; Johnson, D. W., Chem. Soc. Rev. 2014, 43, 1825-1834; (b) Takezawa, Y.; Yoneda, S.; Duprey, J.-L. H. A.; Nakama, T.; Shionoya, M., Chem. Sci. 2016, 7, 3006-3010.

11. (a) Chen, S.; Chen, L.-J.; Yang, H.-B.; Tian, H.; Zhu, W., J. Am. Chem. Soc. 2012, 134, 13596-13599; (b) Han, M.; Luo, Y.; Damaschke, B.; Gómez, L.; Ribas, X.; Jose, A.; Peretzki, P.; Seibt, M.; Clever, G. H., Angew. Chem., Int. Ed. 2016, 55, 445-449.

12. (a) Cullen, W.; Hunter, C. A.; Ward, M. D., Inorg. Chem. 2015, 54, 2626-2637; (b) Frischmann, P. D.; Kunz, V.; Würthner, F., Angew. Chem., Int. Ed. 2015, 54, 7285-7289; (c) Xie, T. Z.; Guo, K.; Guo, Z.; Gao, W. Y.; Wojtas, L.; Ning, G. H.; Huang, M.; Lu, X.; Li, J. Y.; Liao, S. Y.; Chen, Y. S.; Moorefield, C. N.; Saunders, M. J.; Cheng, S. Z. D.; Wesdemiotis, C.; Newkome, G. R., Angew. Chem., Int. Ed. 2015, 54, 9224-9229.
13. Roberts, D. A.; Pilgrim, B. S.; Nitschke, J. R., Chem. Soc. Rev. 2018, 47, 626-644.

14. (a) Zhao, L.; Northrop, B. H.; Stang, P. J., J. Am. Chem. Soc. 2008, 130, 11886-11888; (b) Ronson, T. K.; Pilgrim, B. S.; Nitschke, J. R., J. Am. Chem. Soc. 2016, 138, 10417-10420.

15. (a) Zhao, D.; Tan, S.; Yuan, D.; Lu, W.; Rezenom Yohannes, H.; Jiang, H.; Wang, L. Q.; Zhou, H. C., Adv. Mater. 2010, 23, 90-93; (b) Wang, M.; Lan, W.-J.; Zheng, Y.-R.; Cook, T. R.; White, H. S.; Stang, P. J., J. Am. Chem. Soc. 2011, 133, 10752-10755; (c) Chakrabarty, R.; Stang, P. J., J. Am. Chem. Soc. 2012, 134, 14738-14741; (d) Schneider, M. W.; Oppel, I. M.; Griffin, A.; Mastalerz, M., Angew. Chem., Int. Ed. 2013, 52, 3611-3615; (e) Young, M. C.; Johnson, A. M.; Hooley, R. J., Chem. Commun. 2014, 50, 1378-1380; (f) Brega, V.; Zeller, M.; He, Y.; Peter Lu, H.; Klosterman, J. K., Chem. Commun. 2015, 51, 5077-5080; (g) Holloway, L. R.; Bogie, P. M.; Lyon, Y.; Julian, R. R.; Hooley, R. J., Inorg. Chem. 2017, 56, 11435-11442; (h) Pilgrim, B. S.; Roberts, D. A.; Lohr, T. G.; Ronson, T. K.; Nitschke, J. R., Nat. Chem. 2017, 9, 12761281; (i) Zheng, W.; Yang, G.; Shao, N.; Chen, L.-J.; Ou, B.; Jiang, S.T.; Chen, G.; Yang, H.-B., J. Am. Chem. Soc. 2017, 139, 13811-13820; (j) Low, H.; Mena-Osteritz, E.; von Delius, M., Chem. Sci. 2018, 9, 47854793.

16. (a) Liu, M.; Little, M. A.; Jelfs, K. E.; Jones, J. T. A.; Schmidtmann, M.; Chong, S. Y.; Hasell, T.; Cooper, A. I., J. Am. Chem. Soc. 2014, 136, 7583-7586; (b) Roberts, D. A.; Castilla, A. M.; Ronson, T. K.; Nitschke, J. R., J. Am. Chem. Soc. 2014, 136, 8201-8204; (c) Burke, M. J.; Nichol, G. S.; Lusby, P. J., J. Am. Chem. Soc. 2016, 138, 9308-9315; (d) Lee, S.; Yang, A.; Moneypenny, T. P.; Moore, J. S., J. Am. Chem. Soc. 2016, 138, 2182-2185.

17. (a) Marcos, V.; Stephens, A. J.; Jaramillo-Garcia, J.; Nussbaumer, A. L.; Woltering, S. L.; Valero, A.; Lemonnier, J.-F.; Vitorica-Yrezabal, I. J.; Leigh, D. A., Science 2016, 352, 1555; (b) Lewis, J. E. M.; Beer, P. D.; Loeb, S. J.; Goldup, S. M., Chem. Soc. Rev. 2017, 46, 2577-2591; (c) Cremers, J.; Haver, R.; Rickhaus, M.; Gong, J. Q.; Favereau, L.; Peeks, M. D.; Claridge, T. D. W.; Herz, L. M.; Anderson, H. L., J. Am. Chem. Soc. 2018, 140, 5352-5355.

18. Roberts, D. A.; Pilgrim, B. S.; Cooper, J. D.; Ronson, T. K.; Zarra, S.; Nitschke, J. R., J. Am. Chem. Soc. 2015, 137, 10068-10071.

19. Brooker, S., Chem. Soc. Rev. 2015, 44, 2880-2892.

20. (a) Scherer, M.; Caulder, D. L.; Johnson, D. W.; Raymond, K. N., Angew. Chem., Int. Ed. 1999, 38, 1587-1592; (b) Glasson, C. R. K.; Meehan, G. V.; Davies, M.; Motti, C. A.; Clegg, J. K.; Lindoy, L. F., Inorg. Chem. 2015, 54, 6986-6992.

21. Ousaka, N.; Grunder, S.; Castilla, A. M.; Whalley, A. C.; Stoddart, J. F.; Nitschke, J. R., J. Am. Chem. Soc. 2012, 134, 15528-15537.

22. Bogie, P. M.; Holloway, L. R.; Lyon, Y.; Onishi, N. C.; Beran, G. J. O.; Julian, R. R.; Hooley, R. J., Inorg. Chem. 2018, 57, 4155-4163.

23. (a) Hay, B. P.; Custelcean, R., Cryst. Growth Des. 2009, 9, 2539 2545; (b) Custelcean, R., Chem. Soc. Rev. 2014, 43, 1813-1824.

24. Riddell, I. A.; Hristova, Y. R.; Clegg, J. K.; Wood, C. S.; Breiner, B.; Nitschke, J. R., J. Am. Chem. Soc. 2013, 135, 2723-2733.

25. Riddell, I. A.; Ronson, T. K.; Clegg, J. K.; Wood, C. S.; Bilbeisi, R. A.; Nitschke, J. R., J. Am. Chem. Soc. 2014, 136, 9491-9498.

26. (a) Dawson, R. E.; Hennig, A.; Weimann, D. P.; Emery, D.; Ravikumar, V.; Montenegro, J.; Takeuchi, T.; Gabutti, S.; Mayor, M.; Mareda, J.; Schalley, C. A.; Matile, S., Nat. Chem. 2010, 2, 533; (b) Chifotides, H. T.; Dunbar, K. R., Acc. Chem. Res. 2013, 46, 894-906.

27. Kieffer, M.; Pilgrim, B. S.; Ronson, T. K.; Roberts, D. A.; Aleksanyan, M.; Nitschke, J. R., J. Am. Chem. Soc. 2016, 138, 6813-6821.

28. Ji, Q.; Lirag, R. C.; Miljanic, O. S., Chem. Soc. Rev. 2014, 43, 1873 1884.

29. Shyshov, O.; Brachvogel, R. C.; Bachmann, T.; Srikantharajah, R.; Segets, D.; Hampel, F.; Puchta, R.; Delius, M. v., Angew. Chem., Int. Ed. 2017, 56, 776-781.

30. Pluth, M. D.; Raymond, K. N., Chem. Soc. Rev. 2007, 36, 161-171. 
31. Faulkner, A. D.; Kaner, R. A.; Abdallah, Q. M. A.; Clarkson, G.; Fox, D. J.; Gurnani, P.; Howson, S. E.; Phillips, R. M.; Roper, D. I.; Simpson, D. H.; Scott, P., Nat. Chem. 2014, 6, 797.

32. Haynes, C. J. E.; Zhu, J.; Chimerel, C.; Hernández-Ainsa, S.; Riddell, I. A.; Ronson, T. K.; Keyser, U. F.; Nitschke, J. R., Angew. Chem., Int. Ed. 2017, 56, 15388-15392.

33. Holloway, L. R.; McGarraugh, H. H.; Young, M. C.; Sontising, W.; Beran, G. J. O.; Hooley, R. J., Chem. Sci. 2016, 7, 4423-4427. 


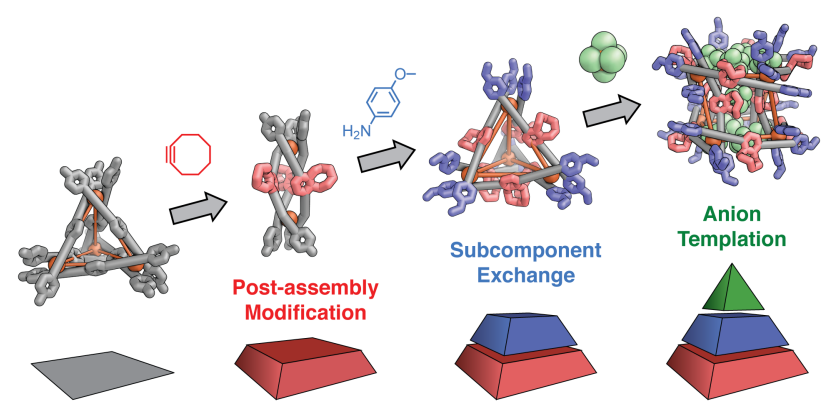

